Article

\title{
Energy-Efficient Subcarrier Pairing and Power Allocation for DF Relay Networks with an Eavesdropper
}

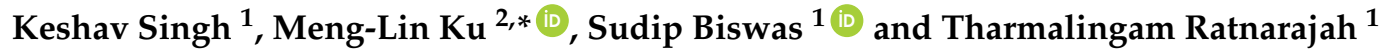 \\ 1 IDCOM, School of Engineering, University of Edinburgh, Edinburgh EH9 3JL, UK; K.Singh@ed.ac.uk (K.S.); \\ Sudip.Biswas@ed.ac.uk (S.B.); T.Ratnarajah@ed.ac.uk (T.R.) \\ 2 Department of Communication Engineering, National Central University, Taoyuan 32001, Taiwan \\ * Correspondence: mlku@ce.ncu.edu.tw; Tel.: +886-3-4227151 (ext. 35512)
}

Received: 13 October 2017; Accepted: 17 November 2017; Published: 24 November 2017

\begin{abstract}
Algorithms for joint subcarrier pairing and power allocation are investigated in order to maximize the worst-case energy efficiency (EE) in dual-hop decode-and-forward (DF) relay networks in the presence of an active eavesdropper. Accordingly, we study the impact of number of subcarriers on the trade-off in performance between the EE and the spectrum efficiency (SE). The formulated EE optimization problem is the ratio of the secure SE over the entire power consumption in the network, subject to the constraints of total transmit power and subcarrier pairing. A near-optimal iterative algorithm is proposed to perform the subcarrier pairing and power allocation for achieving the maximum EE in the networks. Furthermore, a suboptimal algorithm is proposed with two-step resource allocation. By considering the subcarrier channel quality of the source-to-relay and relay-to-destination links, the subcarrier pairing is first performed, followed by an energy-efficient iterative power allocation scheme to maximize the EE. Numerical results validate the effectiveness and correctness of the proposed algorithms.
\end{abstract}

Keywords: power allocation; subcarrier pairing; decode-and-forward; energy efficiency; eavesdropper; relay networks

\section{Introduction}

The enormous growth of energy consumption in wireless communication networks has recently drawn significant attention from both academics and industry. The energy consumption in telecommunication networks is predicted to surge rapidly due to the increasing escalation of data rates and the use of numerous smart devices [1]. Improving the energy efficiency (EE) of networks is of paramount importance in striving towards green 5G radio [2]. Accordingly, investigation on energy-aware system design and power and subcarrier allocation techniques that provide significant energy savings [3] is inevitable in the research of future wireless communications systems.

Cooperative communications have been proven to be promising technologies for enhancing the throughput, extending the coverage, and enhancing the link reliability. Various relaying protocols have been proposed, among which amplify-and-forward (AF) and decode-and-forward (DF) are two main protocols [4]. In the AF protocol, the relay retransmits the amplified signal to the destination, while in the DF protocol, the relay decodes the received signals and retransmits the re-encoded information bits to the destination. While in terms of implementation complexity the AF protocol has an advantage over others, when the channel quality of the source-to-relay (SR) link is good, the DF protocol performs better than the AF protocol. Furthermore, different channel coding can be used at the source and the relay nodes for the DF protocol, which is another advantage of it. 
With regard to the power consumption in wireless networks, dynamic power consumption due to transmit power, which is allocated in response to the instantaneous channel conditions, and static power consumption due to signal processing at each node, site cooling, battery backup, etc., are the two main sources of power dissipation [5]. Further, for DF relay networks, the EE performance is highly dependent on these two sources [6]. Accordingly, in this paper, through a joint optimization of the subcarrier pairing and transmit power allocation, we will maximize the EE of a dual-hop DF relay network in the presence of an eavesdropper. Of late, resource allocation has been studied in literature for enhancing the throughput of DF relay networks. In $[7,8]$, the optimal power allocation schemes were investigated to maximize the rate in DF networks under total transmit power or individual power constraints. Similarly, the authors in $[9,10]$ studied the optimization problems for joint subcarrier pairing and power allocation with a total network power constraint or with individual power constraints for the source and the relay nodes. In particular, to overcome the interference problem, the authors in [10] performed a joint optimization of relay selection, power allocation, and subcarrier assignment. Further, in these existing works [7-11], the throughput maximization was the main design goal. However, similar resource allocation schemes cannot be directly used when the network EE is considered to be the objective function. In this regard, in [12], an energy-efficient resource allocation algorithm was proposed for downlink network with a large number of transmit antennas. However, instead of worst-case EE maximization, the authors of [12] focused on maximizing the total EE for downlink networks. Moreover, the scheme described in [12] cannot be easily applied to multi-hop scenarios.

Additionally, due to the broadcasting nature of wireless channels, a secure exchange of information between mobile users is required. In [13], a secure cooperative communications scheme was investigated for cognitive radio networks, and a joint destination-aided cooperative jamming and precoding technique for dual-hop AF MIMO relay networks was proposed. Further, Yang et al. in [14] comprehensively analyzed secrecy performance for multiuser regenerative relay wiretap networks, while the authors in [15] proposed an artificial-noise (AN)-aided relay selection scheme to enhance the security of DF relay networks. The power allocation scheme is investigated in [16] for maximizing the secure energy efficiency (SEE) for the DF relay network, but only for a single subcarrier.

Considering the aforementioned discussions, in this paper we jointly investigate both secure transmission and EE maximization issues for a dual-hop DF relay network, which consists of one source node, one destination node, and one relay node with an eavesdropper between the relay node and the destination node. The key contributions are highlighted as follows:

- In contrast to [13-17], where the secure sum rate/EE maximization for a single subcarrier is served as the main design goal, in this paper, a joint optimization problem is formulated to maximize the secure EE of the network, subject to a total transmit power budget. The original optimization problem is NP-hard. We first transform the secure EE problem into an equivalent convex problem for finding the optimal power allocation under given subcarrier pairing. The optimization problem then becomes a mixed-integer binary non-linear programming problem. Instead of searching over all possible subcarrier pairing combinations, a near-optimal EE maximization (EEM) algorithm is found by using the Hungarian method to reduce the computational burden. To get more insights, we derive a closed-form expression for the source and the relay power allocation.

- Furthermore, a suboptimal algorithm with even less complexity but acceptable performance degradation is investigated. Next, we analyze the complexity of the proposed algorithms, and evaluate the performance and compare with an EEM algorithm without an eavesdropper (EEM w/o E) by computer simulation for understanding the trade-off between them.

The organization of this paper is as follows. The system model and problem formulation are described in Section 2. A near-optimal subcarrier pairing and power allocation algorithm for maximizing the EE is presented in Section 3, whereas the suboptimal algorithm is illustrated in Section 4. The complexity analysis is presented in Section 5, followed by the numerical results in Section 6. Finally, Section 7 concludes the paper. 


\section{System Model and Problem Formulation}

\subsection{System Model}

We consider a dual-hop DF relay network as shown in Figure 1, with a single source-destination pair $(S-D)$, a single relay node $(R)$, and an eavesdropper node $(E)$ between the relay node and the destination node. We assume that all the nodes have a single antenna and full channel state information (CSI) knowledge by performing conventional pilot-based channel estimation methods. By utilizing the channel reciprocity scheme between forward and backward transmissions through orthogonal pilot signals, the CSIs can be estimated at the relay and the destination nodes. The reciprocity is practical as long as the coherence time of the channel is larger than the dual-hop communication time. The destination then feeds back the estimated CSI to the relay. Due to the path-loss and large-scale fading, the direct link between the source and the destination nodes is ignored. Moreover, the relay node is operated in a half-duplex mode with two transmission phases. The eavesdropper can overhear the information transmitted by the relay node. In the first hop, the source node sends signals to the relay node, whereas the relay re-transmits the re-encoded signals to the destination node during the second hop. In the meantime, the source node stays ideal. The eavesdropper eavesdrops the transmitted messages in the second time slot. Moreover, the SR and relay-to-destination (RD) channels on any subcarrier are assumed to be frequency-flat.

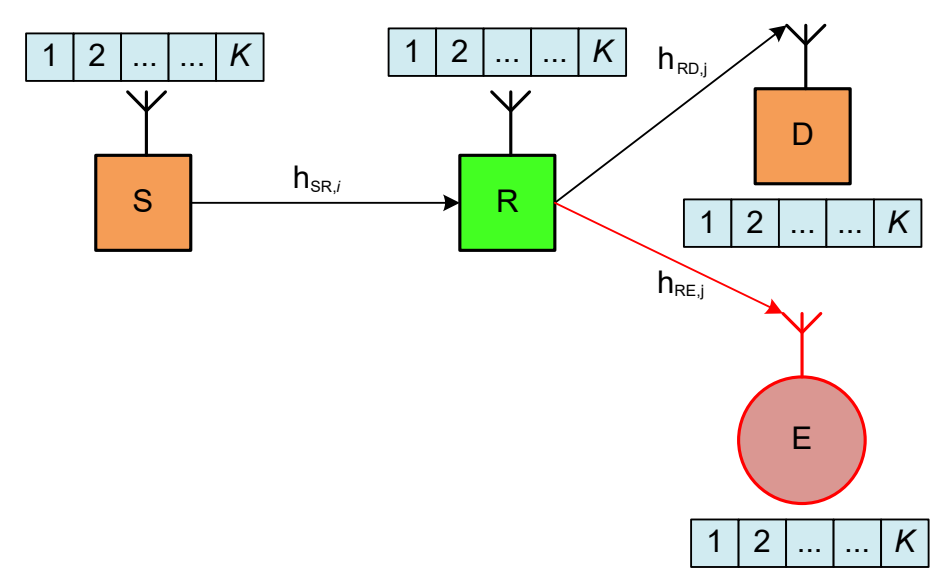

Figure 1. A decode-and-forward (DF) relay network with an eavesdropper.

Let $\mathcal{K}=\{1,2, \cdots, K\}$ denote a set of subcarriers within the available bandwidth. The channel coefficient of the SR link on the $i$ th subcarrier, $i \in \mathcal{K}$, is denoted by $h_{S R, i}$, and that of the RD link on the $j$ th subcarrier, $j \in \mathcal{K}$, is expressed as $h_{R D, j}$. Further, the channel coefficient for the relay-to-eavesdropper (RE) link on the $j$ th subcarrier is given by $h_{R E, j}$.

The signal received by the relay node in the first phase on the $i$ th subcarrier is written as

$$
y_{R, i}=\sqrt{P_{S, i}} h_{S R, i} s_{i}+n_{R, i}
$$

where $s_{i}$ is the transmitted signal from the source node on the $i$ th subcarrier with unit power, i.e., $E\left[\left|s_{i}\right|^{2}\right]=1, P_{S, i}$ indicates its transmit power, and $n_{R, i}$ denotes zero-mean Gaussian noise at the relay node with variance $\sigma_{R, i}^{2}$. Using (1), the signal-to-noise power ratio (SNR) at the relay node is

$$
\Gamma_{S R, i}=\frac{P_{S, i}\left|h_{S R, i}\right|^{2}}{\sigma_{R, i}^{2}}
$$


Supposing that the relay node decodes the data symbol perfectly, in the second phase, the received signal at the destination node and the eavesdropper on the $j$ th subcarrier can be expressed as

$$
\begin{aligned}
& y_{D, j}=\sqrt{P_{R, j}} h_{R D, j} s_{j}+n_{D, j} \\
& y_{E, j}=\sqrt{P_{R, j}} h_{R E, j} s_{j}+n_{E, j}
\end{aligned}
$$

where $n_{D, j}$ and $n_{E, j}$ are defined similar to $n_{R, i}$ but with variance $\sigma_{D, j}^{2}$ and $\sigma_{E, j}^{2}$, respectively. Using (3) and (4), the SNR at the destination and the eavesdropper can be written as

$$
\begin{gathered}
\Gamma_{R D, j}=\frac{P_{R, j}\left|h_{R D, j}\right|^{2}}{\sigma_{D, j}^{2}} \\
\Gamma_{R E, j}=\frac{P_{R, j}\left|h_{R E, j}\right|^{2}}{\sigma_{E, j}^{2}}
\end{gathered}
$$

The secrecy rate $R_{\text {sec }}(i, j)$ with the pairing of the $i$ th subcarrier for the SR link and the $j$ th subcarrier for the RD link is given by

$$
R_{s e c}(i, j)=\left[\frac{1}{2} \log _{2}\left(1+\min \left\{\Gamma_{S R, i}, \Gamma_{R D, j}\right\}\right)-\log _{2}\left(1+\Gamma_{R E, j}\right)\right]^{+}
$$

where $[\cdot]^{+}=\max \{0, \cdot\}$ and $1 / 2$ is due to the fact that transmission takes place in two hops. Further, both the transmit and static power dissipation are taken into account to design energy-efficient relay networks. The transmit power relies upon instantaneous channel coefficients, while the static power involves the processing and circuit power. Therefore, we define the total power dissipation in the network as follows [6]:

$$
P_{\text {total }}=\underbrace{\sum_{i=1}^{K} P_{S, i}+P_{S P, S}}_{\text {source power dissipation }}+\underbrace{\sum_{j=1}^{K} P_{R, j}+P_{S P, R}}_{\text {relay power dissipation }}
$$

where $P_{S P, S}$ and $P_{S P, R}$ denote the total static power consumption at the source and the relay nodes, respectively.

\subsection{Optimization Problem Formulation}

The key task of this work is to maximize the EE through the joint optimization of the subcarrier pairing and power allocation. From (7) and (8), the objective function is expressed as

$$
\Sigma_{E}\left(\mathbf{P}_{S}, \mathbf{P}_{R}, \boldsymbol{\rho}\right)=\frac{\frac{1}{2} \sum_{i=1}^{K} \sum_{j=1}^{K} \rho_{i, j} \log _{2}\left(1+\min \left\{\Gamma_{S R, i}, \Gamma_{R D, j}\right\}\right)-\frac{1}{2} \sum_{j=1}^{K} \log _{2}\left(1+\Gamma_{R E, j}\right)}{\underbrace{\sum_{i=1}^{K} \sum_{j=1}^{K} \rho_{i, j}\left(P_{S, i}+P_{R, j}\right)+P_{S C}}_{\triangleq P\left(\mathbf{P}_{S}, \mathbf{P}_{R}, \boldsymbol{\rho}\right)}}
$$

where $\mathbf{P}_{S}=\left[P_{S, 1}, P_{S, 2}, \cdots, P_{S, K}\right]$ and $\mathbf{P}_{R}=\left[P_{R, 1}, P_{R, 2}, \cdots, P_{R, K}\right]$ are the transmit power vectors for the source and the relay nodes, respectively, while $P_{S C}=P_{S P, S}+P_{S P, R}$. The term $\sum_{i=1}^{K} \sum_{j=1}^{K} \rho_{i, j}\left(P_{S, i}+P_{R, j}\right)$ serves as the actual transmit power consumption under subcarrier pairing, and $\rho \in\{0,1\}^{K \times K}$ is a matrix with the entry $\rho_{i, j}$ which represents a subcarrier pairing indicator to guarantee that each subcarrier in the SR hop is only paired with a single subcarrier in the RD hop, i.e., $\rho_{i, j}=1$ when the $i$ th 
subcarrier in the SR hop is paired with the $j$ th subcarrier in the RD hop and $\rho_{i, j}=0$ otherwise. With total transmit power and subcarrier pairing constraints, the secure energy efficiency (EE) maximization problem can be formulated as follows:

$$
\begin{aligned}
\text { (P.1) } \max _{\mathbf{P}_{S}, \mathbf{P}_{R}, \boldsymbol{\rho}} & \Sigma_{E}\left(\mathbf{P}_{S}, \mathbf{P}_{R}, \boldsymbol{\rho}\right) \\
\text { s.t. } & (\text { C.1 }) \quad \sum_{i=1}^{K} \sum_{j=1}^{K} \rho_{i, j}\left(P_{S, i}+P_{R, j}\right) \leq P_{\max } ; \\
& \left(\text { C.2) } \quad \sum_{j=1}^{K} \rho_{i, j}=1, \forall i ;\right. \\
& (\text { C.3 }) \quad \rho_{i, j} \in\{0,1\}, \forall i, j ; \\
& \text { (C.4) } P_{S, i} \geq 0, P_{R, j} \geq 0, \forall i, j .
\end{aligned}
$$

where the objective function $\Sigma_{E}\left(\mathbf{P}_{S}, \mathbf{P}_{R}, \boldsymbol{\rho}\right)$ denotes the secure EE and the constraint (C.1) presents the entire power consumption under the subcarrier paring condition with a maximum transmit power budget $P_{\max }$ for the source and the relay nodes. Here, the subcarrier pairing constraint (C.2) ensures that each subcarrier in the first hop is only paired with a single subcarrier in the second hop, and vice versa, while (C.3) stipulates that the subcarrier pairing variables take binary values. The constraint (C.4) makes the transmit power values of the source and the relay nodes non-negative.

\section{Energy-Efficient Subcarrier Pairing and Power Allocation Algorithm}

The objective function in (33) is in a fractional form and the optimization problem is mixed-integer binary non-linear programming. In result, the problem becomes non-convex [18]. To make the problem tractable, an auxiliary variable $\Omega_{i}, i \in\{1, \ldots, K\}$ is introduced and change of variables $\hat{P}_{S, i}=\ln P_{S, i}, \hat{P}_{R, j}=\ln P_{R, j}$, and $\hat{\Omega}_{i}=\ln \Omega_{i}$ is applied to transform the problem as follows:

$$
\begin{aligned}
& \text { (P.2) } \max _{\mathbf{P}_{S, \mathbf{P}_{R}, \boldsymbol{\rho}, \Omega}} \frac{\frac{1}{2} \sum_{i=1}^{K} \sum_{j=1}^{K} \rho_{i, j} \log _{2}\left(1+e^{\hat{\Omega}_{i}}\right)-\sum_{j=1}^{K} \log _{2}\left(1+\hat{\Gamma}_{R E, j}\right)}{\sum_{i=1}^{K} \sum_{j=1}^{K} \rho_{i, j}\left(e^{\hat{P}_{S, i}}+e^{\hat{P}_{R, j}}\right)+P_{S C}} \\
& \text { s.t. } \quad(C .1) \quad \sum_{i=1}^{K} \sum_{j=1}^{K} \rho_{i, j}\left(e^{\hat{P}_{S, i}}+e^{\hat{P}_{R, j}}\right) \leq P_{\max } \\
& (\text { C.2) }-(C .4) \text {; } \\
& \text { (C.5) } e^{\hat{\Omega}_{i}-\hat{P}_{S, i}} \frac{\sigma_{R, i}^{2}}{\left|h_{S R, i}\right|^{2}} \leq 1, \quad \forall i \\
& \text { (C.6) } e^{\hat{\Omega}_{j}-\hat{P}_{R, j}} \frac{\sigma_{D, j}^{2}}{\left|h_{R D, j}\right|^{2}} \leq 1, \quad \forall j .
\end{aligned}
$$

where $\Omega=\left[\Omega_{1}, \Omega_{2}, \cdots, \Omega_{K}\right]$ and $\hat{\Gamma}_{R E, j}$ can be given as

$$
\hat{\Gamma}_{R E, j}=\frac{e^{\hat{P}_{R, j}}\left|h_{R E, j}\right|^{2}}{\sigma_{E, j}^{2}}, \quad \forall j
$$

To deal with the fractional objective function, we now transform the objective function in (11) into a subtractive form by using a successive convex approximation (SCA) method to impose two lower bounds as follows: 


$$
\begin{aligned}
\log _{2}\left(1+e^{\hat{\Omega}_{i}}\right) & \geq \frac{\alpha_{i}^{d}}{\ln 2} \hat{\Omega}_{i}+\beta_{i}^{d}, \forall i \\
\log _{2}\left(1+\hat{\Gamma}_{R E, j}\right) & \geq \frac{\alpha_{j}^{e}}{\ln 2}\left(\hat{P}_{R, j}+\ln \left(\left|h_{R E, j}\right|^{2}\right)-\ln \left(\sigma_{E, j}^{2}\right)\right)+\beta_{j}^{e}, \forall j
\end{aligned}
$$

where the coefficients in (13) are determined in the following manner [6]:

$$
\begin{aligned}
\alpha_{i}^{d} & =\frac{\xi_{i}}{1+\xi_{i}} \\
\beta_{i}^{d} & =\log _{2}\left(1+\xi_{i}\right)-\alpha_{i}^{d} \log _{2}\left(\xi_{i}\right)
\end{aligned}
$$

for any $\xi_{i}>0$. Note that the equality in (13) holds when $\alpha_{i}^{d}=\hat{\Omega}_{i} / 1+\hat{\Omega}_{i}$ and $\beta_{i}^{d}=\log _{2}\left(1+\hat{\Omega}_{i}\right)-\alpha_{i}^{d} \log _{2}\left(\hat{\Omega}_{i}\right)$, and the equality holds for $\left(\alpha_{i}^{d}, \beta_{i}^{d}\right)=(0,1)$ if $\hat{\Omega}_{i} \rightarrow \infty$. Also, $\alpha_{j}^{e}$ and $\beta_{j}^{e}$ are defined similarly. After substituting (13) and (14) into (11) the objective function turns out to be concave-over-convex and thus we apply the well-known Dinkelbach method to transform it into a subtractive form [19]:

$$
\begin{aligned}
\text { (P.3) } \max _{\mathbf{P}_{S}, \mathbf{P}_{R}, \rho, \Omega} & \frac{1}{2} \sum_{i=1}^{K} \sum_{j=1}^{K} \rho_{i, j}\left(\frac{\alpha_{i}^{d}}{\ln 2} \hat{\Omega}_{i}+\beta_{i}^{d}\right) \\
- & -\sum_{j=1}^{K} \frac{\alpha_{j}^{e}}{\ln 2}\left(\hat{P}_{R, j}+\ln \left(\left|h_{R E, j}\right|^{2}\right)-\ln \left(\sum_{i=1}^{2} \sum_{j=1}^{K} \rho_{i, j}\left(e^{\hat{P}_{S, i}}+e^{\hat{P}_{R, j}}\right)+P_{S C}\right)\right. \\
\text { s.t. } \quad(\text { C.1 })-(\text { C.6 }) &
\end{aligned}
$$

where $\Psi(t)$ is a positive parameter at iteration $t$ and it is treated as a penalty factor for the power utilization. When the parameter approaches zero, it indicates that the penalty is zero for the power utilization, and the resource allocation problem converts into a problem of secure sum rate maximization in a relay network. However, for another extreme case where $\Psi(t)$ approaches infinity, no transmission is the best policy to maximize the objective function in (17).

We first discuss the optimal solution for a given parameter $\Psi(t)$. For simplicity, we use $\Psi$ instead of $\Psi(t)$ in the remaining parts of this paper. Since the problem (P.3) is a mixed-binary integer non-linear programming problem, the computational complexity is very high for finding the globally optimal solution. For a fixed subcarrier pairing in (17), the problem (P.3) becomes a standard convex optimization problem, and the optimal solution can be determined by applying the dual decomposition method.

The Lagrangian function of (17) can be given by

$$
\begin{aligned}
\mathcal{L}\left(\mathbf{P}_{S}, \mathbf{P}_{R}, \boldsymbol{\rho}, \boldsymbol{\Omega}, \lambda,\right. & \boldsymbol{\mu}, \boldsymbol{v})=\frac{1}{2} \sum_{i=1}^{K} \sum_{j=1}^{K} \rho_{i, j}\left(\frac{\alpha_{i}^{d}}{\ln 2} \hat{\Omega}_{i}+\beta_{i}^{d}\right)-\sum_{j=1}^{K} \frac{\alpha_{j}^{e}}{\ln 2}\left(\hat{P}_{R, j}+\ln \left(\left|h_{R E, j}\right|^{2}\right)-\ln \left(\sigma_{E, j}^{2}\right)\right)-\beta_{j}^{e} \\
& -\Psi\left(\sum_{i=1}^{K} \sum_{j=1}^{K} \rho_{i, j}\left(e^{\hat{P}_{S, i}}+e^{\hat{P}_{R, j}}\right)+P_{S C}\right)-\lambda\left(\sum_{i=1}^{K} \sum_{j=1}^{K} \rho_{i, j}\left(e^{\hat{P}_{S, i}}+e^{\hat{P}_{R, j}}\right)-P_{\max }\right) \\
& -\sum_{i=1}^{K} \mu_{i}\left(e^{\hat{\Omega}_{i}-\hat{P}_{S, i}} \frac{\sigma_{R, i}^{2}}{\left|h_{S R, i}\right|^{2}}-1\right)-\sum_{j=1}^{K} v_{j}\left(e^{\hat{\Omega}_{j}-\hat{P}_{R, j}} \frac{\sigma_{D, j}^{2}}{\left|h_{R D, j}\right|^{2}}-1\right)
\end{aligned}
$$

where $\lambda \geq 0$ is a Lagrangian dual variable associated with the transmit power constraint. The vectors $\boldsymbol{\mu}=\left[\mu_{1}, \mu_{2}, \cdots, \mu_{K}\right]^{T}$ and $\boldsymbol{v}=\left[v_{1}, v_{2}, \cdots, v_{K}\right]^{T}$ are the dual variables corresponding to the constraints (C.5) and (C.6), respectively. Then, the dual Lagrangian function can be written as

$$
g(\lambda, \boldsymbol{\mu}, \boldsymbol{v}) \triangleq \max _{\mathbf{P}_{S}, \mathbf{P}_{R}, \boldsymbol{\rho}, \boldsymbol{\Omega}} \mathcal{L}\left(\mathbf{P}_{S}, \mathbf{P}_{R}, \boldsymbol{\rho}, \boldsymbol{\Omega}, \lambda, \boldsymbol{\mu}, \boldsymbol{v}\right)
$$


and the dual optimization problem is

$$
\min _{\lambda, \mu, v \geq 0} g(\lambda, \mu, v)
$$

This dual problem is divided into a master problem and a subproblem, and an iterative approach is adopted to solve it. In the subproblem, the $\mathbf{P}_{S}, \mathbf{P}_{R}$, and $\boldsymbol{\rho}$ are obtained for fixed Lagrangian multipliers, whereas by resolving the master problem, we update $\lambda, \mu$, and $\nu$.

\subsection{Solution of the Subproblem}

By employing the Karush-Kuhn-Tucker (KKT) conditions, we can find the optimal power allocation for a given subcarrier pairing $(i, j)$. To find the optimal $P_{R, j}^{(t+1)^{\star}}$ and $\Omega_{i}^{(t+1)^{\star}}$ in iteration $(t+1)$, we take the partial derivative of (18) with respect to $P_{R, j}$ and $\Omega_{i}$ and equate the results to zero as

$$
\begin{aligned}
& P_{R, j}^{(t+1)^{\star}}=\left[\left(\frac{v_{j} e^{\hat{\Omega}_{j}} \sigma_{D, j}^{2}}{\left|h_{R D, j}\right|^{2}\left(\frac{\alpha_{j}^{e}}{\ln 2}-(\Psi+\lambda) \sum_{i=1}^{K} \rho_{i, j}\right)}\right)^{\frac{1}{2}}\right]^{+} \\
& \Omega_{i}^{(t+1)^{\star}}=\left[\frac{\frac{\alpha_{i}^{d}}{2 \ln 2} \sum_{j=1}^{K} \rho_{i, j}}{\frac{\mu_{i} \sigma_{R, i}^{2}}{e^{\hat{P}_{S, i}}\left|h_{S R, i}\right|^{2}}-\frac{v_{i} \sigma_{D, i}^{2}}{e^{\hat{P}_{R, i}}\left|h_{R D, i}\right|^{2}}}\right]^{+}
\end{aligned}
$$

The source power at the $(t+1)^{t h}$ iteration, $P_{S, i}^{(t+1)}$, can be updated through the subgradient method as

$$
P_{S, i}^{(t+1)^{\star}}=\left[P_{S, i}^{(t)}+\varepsilon_{1}^{(t)}\left(\frac{\mu_{i}^{(t)} \sigma_{R, i}^{(t)^{2}}}{\left|h_{S R, i}\right|^{(t)^{2}}}+\left(\Psi^{(t)}+\lambda^{(t)}\right) \sum_{j=1}^{K} \rho_{i, j}^{(t)}\right)\right]^{+}
$$

where $\varepsilon_{1}^{(t)}$ is a positive step size. To determine the optimal subcarrier pairing, we put (23) and (21) into (18), yielding

$$
\mathcal{L}\left(\mathbf{P}_{S}^{\star}, \mathbf{P}_{R}^{\star}, \boldsymbol{\rho}, \mathbf{\Omega}^{\star}, \lambda, \boldsymbol{\mu}, \boldsymbol{v}\right)=\sum_{i=1}^{K} \sum_{j=1}^{K} \rho_{i, j} Y_{i, j}+Z(\lambda, \boldsymbol{\mu}, \boldsymbol{v})
$$

where

$$
\begin{gathered}
Y_{i, j}=\frac{\alpha_{i}^{d}}{2 \ln 2} \ln \Omega_{i}^{\star}+\frac{\beta_{i}^{d}}{2}-(\Psi+\lambda)\left(P_{S, i}^{\star}+P_{R, j}^{\star}\right) \\
Z(\lambda, \mu, v)=-\sum_{j=1}^{K} \frac{\alpha_{j}^{e}}{\ln 2}\left(\ln P_{R, j}^{\star}+\ln \left(\left|h_{R E, j}\right|^{2}\right)-\ln \left(\sigma_{E, j}^{2}\right)\right)-\beta_{j}^{e}-\Psi P_{S C}+\lambda P_{\max } \\
-\sum_{i=1}^{K} \mu_{i}\left(\frac{\Omega_{i}^{\star} \sigma_{R, i}^{2}}{P_{S, i}^{\star}\left|h_{S R, i}\right|^{2}}-1\right)-\sum_{j=1}^{K} v_{j}\left(\frac{\Omega_{j}^{\star} \sigma_{D, j}^{2}}{P_{R, j}^{\star}\left|h_{R D, j}\right|^{2}}-1\right)
\end{gathered}
$$


The first term in $Y_{i, j}$ denotes the sum rate obtained for the given subcarrier pairing $(i, j)$, while the remaining term $Z(\lambda, \mu, v)$, which is always independent of $\rho_{i, j}$, works as a penalty for the power dissipation.

As a result, for the optimal power allocation $\left(\mathbf{P}_{R}^{\star}, \mathbf{P}_{S}^{\star}\right)$ derived in (21) and (23), the optimal subcarrier pairing $\boldsymbol{\rho}^{\star}$ can be found by solving the problem (P.4) as follows:

$$
\begin{array}{ll}
\text { (P.4) } \max _{\boldsymbol{\rho}} \sum_{i=1}^{K} \sum_{j=1}^{K} \rho_{i, j} Y_{i, j} \\
\text { s.t. } \quad(\text { C.2) \& (C.3) }
\end{array}
$$

Define a $K \times K$ matrix $\mathbf{Y}$ as follows:

$$
\mathbf{Y}=\left[\begin{array}{ccc}
Y_{1,1} & \cdots & Y_{1, K} \\
\vdots & \ddots & \vdots \\
Y_{K, 1} & \cdots & Y_{K, K}
\end{array}\right]
$$

The optimal subcarrier pairing $\rho^{\star}$ can be determined by choosing exactly one value in each row and column of the matrix $\mathbf{Y}$ with the help of the Hungarian method [20] such that the sum of these $K$ values is maximized.

\subsection{Solution of the Master Problem}

Using the subgradient method, the dual variables $\lambda, \mu$, and $v$ can be iteratively updated as

$$
\begin{aligned}
& \lambda^{(l+1)}=\left[\lambda^{(l)}-\varepsilon_{2}^{(l)}\left(P_{\max }-\sum_{i=1}^{K} \sum_{j=1}^{K} \rho_{i, j}^{\star}\left(P_{S, i}^{\star}+P_{R, j}^{\star}\right)\right)\right]^{+} \\
& \mu_{i}^{(l+1)}=\left[\mu_{i}^{(l)}-\varepsilon_{3}^{(l)}\left(1-\frac{\Omega_{i}^{\star} \sigma_{R, i}^{2}}{P_{S, i}^{\star}\left|h_{S R, i}\right|^{2}}\right)\right]^{+} \\
& v_{i}^{(l+1)}=\left[v_{i}^{(l)}-\varepsilon_{4}^{(l)}\left(1-\frac{\Omega_{j}^{\star} \sigma_{D, j}^{2}}{P_{R, j}^{\star}\left|h_{R D, j}\right|^{2}}\right)\right]^{+}
\end{aligned}
$$

where $\varepsilon_{i}^{(l)}, i \in\{2,3,4\}$, are positive step sizes. The parameter $\Psi(t)$ can be updated as [6]:

$$
\Psi(t)=\frac{\frac{1}{2} \sum_{i=1}^{K} \sum_{j=1}^{K} \rho_{i, j}\left(\frac{\alpha_{i}^{d}}{\ln 2} \ln \Omega_{i}^{\star}+\beta_{i}^{d}\right)-\sum_{j=1}^{K} \frac{\alpha_{j}^{e}}{\ln 2}\left(\ln P_{R, j}^{\star}+\ln \left(\left|h_{R E, j}\right|^{2}\right)-\ln \left(\sigma_{E, j}^{2}\right)\right)-\beta_{j}^{e}}{\left(\sum_{i=1}^{K} \sum_{j=1}^{K} \rho_{i, j}\left(e^{\hat{P}_{S, i}}+e^{\hat{P}_{R, j}}\right)+P_{S C}\right)}
$$

The $\Psi$-based iterative EEM algorithm for joint power allocation and subcarrier pairing is finally summarized in Algorithm 1. 


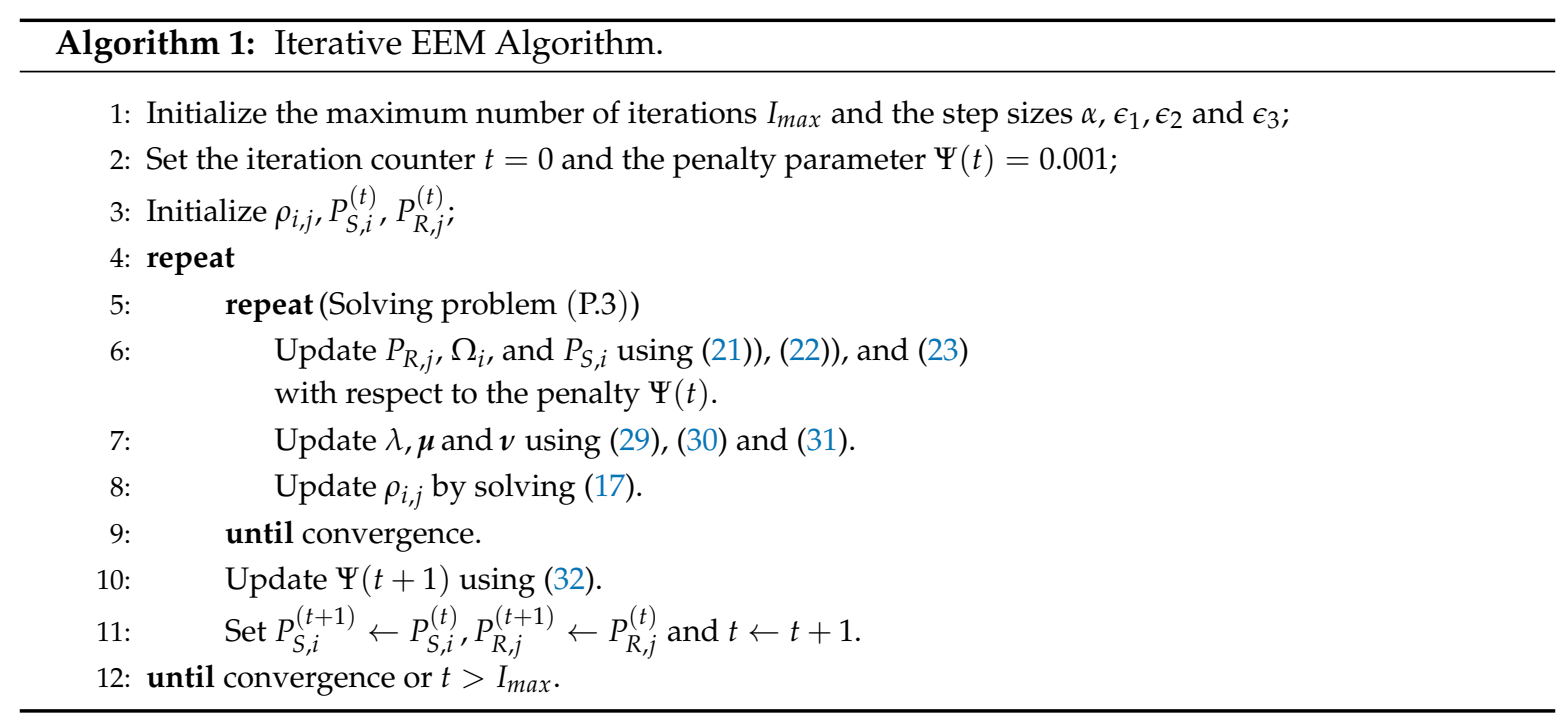

The quality-of-service (QoS)-based joint subcarrier pairing and power allocation design for maximizing the secrecy EE can be formulated as:

$$
\begin{array}{cl}
\max _{\mathbf{P}_{S}, \mathbf{P}_{R}, \boldsymbol{\rho}} & \Sigma_{E}\left(\mathbf{P}_{S}, \mathbf{P}_{R}, \boldsymbol{\rho}\right) \\
\text { s.t. } & (C .1) \sum_{i=1}^{K} \sum_{j=1}^{K} \rho_{i, j}\left(P_{S, i}+P_{R, j}\right) \leq P_{\max } ; \\
& (C .2) \sum_{j=1}^{K} \rho_{i, j}=1, \quad \forall i ; \\
& (C .3) \rho_{i, j} \in\{0,1\}, \forall i, j ; \\
& (C .4) \frac{1}{2} \log _{2}\left(1+\min \left\{\Gamma_{S R, i}, \Gamma_{R D, j}\right\}\right) \geq R_{(i, j), \min }, \forall(i, j) ; \\
& \left(\text { C.5 } P_{S, i} \geq 0, P_{R, j} \geq 0, \forall i, j .\right.
\end{array}
$$

Note that the constraint (C.4) denotes the quality-of-service (QoS) metric for a user pair on the $(i, j)$ th subcarrier pair. Accordingly, we can resolve the new optimization problem in a same way as the problem in (P.1).

\section{Energy-Efficient Suboptimal Resource Allocation Algorithm}

The complexity of the near-optimal algorithm proposed in Section 3 becomes high when the value of $K$ is large. Therefore, a low-complexity suboptimal algorithm with two steps is investigated in this section, whose EE and SE performance is near that of the near-optimal one. This suboptimal algorithm is elaborated:

1. Intuitively, a higher power value is allocated to a subcarrier pair with better channel quality from the source to the destination nodes. Hence, the subcarriers of the SR and RD links with a higher end-to-end channel gain are paired together with a higher priority. Firstly, the SR subcarriers are organized in a descending order according to their channel gains, and the RD subcarriers are also ordered in similar way. Then, the corresponding subcarriers are paired with each other in sequence. We set $\rho_{i, j}=1$, if the $i$ th subcarrier of the SR link is paired with the $j$ th subcarrier of the RD link; otherwise, $\rho_{i, j}=0$.

2. The next step is to get the optimal power allocation $\left(\mathbf{P}_{S}, \mathbf{P}_{R}\right)$ for maximizing EE by solving (P.3) for the obtained subcarrier pairing $\rho_{i, j}$ in the step 1 . 


\section{Complexity Analysis}

In this section, the complexity analysis is presented to demonstrate the complexity reduced by the near-optimal and the suboptimal algorithms when the penalty factor $\Psi$ converges in $L$ iterations.

We begin with the complexity analysis of the near-optimal EEM algorithm as follows. Due to $K$ subcarriers in each hop, $K^{2}$ subproblems are required to be solved in order to find the optimal solutions of (P.3). Since the near-optimal solution $\left(P_{S, i}^{\star}, P_{R, j}^{\star}, \Omega_{i}^{\star}\right)$ is found with the total power constraint, the complexity is $\mathcal{O}\left(V^{3}+1\right)$, where the maximum power level for the source and the relay nodes on each subcarrier are denoted by $V$. Moreover, finding the optimal subcarrier pairing using the Hungarian produces a complexity of $\mathcal{O}\left(K^{3}\right)$. The complexity due to updating a dual variable is $\mathcal{O}\left(2^{\circ}\right)$ (for example, $\varrho=2$ for the ellipsoid method [21]). Hence, the overall complexity for updating dual variables is $\mathcal{O}\left(2^{2 e}\right)$. Supposing the dual objective function converges in $W$ iterations, the entire complexity for the near-optimal EEM algorithm is $\mathcal{O}\left(2^{2 \varrho} K^{2} W L\left(V^{3}+K+1\right)\right)$.

However, the implementation of the subcarrier pairing in step 1 of suboptimal algorithmin requires a complexity of $\mathcal{O}(2 K)$, while the power allocation has the complexity of $\mathcal{O}\left(K\left(V^{3}+1\right)\right)$ and the total complexity for updating dual variables is $\mathcal{O}\left(2^{2 \varrho}\right)$. If the dual objective function converges in $W^{\prime}$ iterations, the overall complexity for the suboptimal algorithm becomes $\mathcal{O}\left(2^{2 \varrho} \mathrm{KWL}\left(V^{3}+3\right)\right)$ with $W^{\prime}=W$. Finally, the complexity of the optimal exhaustive search (ES) algorithm [22] is given by $\mathcal{O}\left(2^{2 \varrho} W L K !\left(V^{3}+1\right)\right)$. Table 1 presents the summary of the complexity comparison of the three algorithms.

Table 1. Complexity comparison. ES: exhaustive search; EEM: energy efficiency maximization.

\begin{tabular}{cc}
\hline Algorithm & Complexity \\
\hline Optimal ES & $\mathcal{O}\left(2^{2 \varrho} W L K !\left(V^{3}+1\right)\right)$ \\
EEM & $\mathcal{O}\left(2^{2 \varrho} K^{2} W L\left(V^{3}+K+1\right)\right)$ \\
Suboptimal & $\mathcal{O}\left(2^{2 \varrho} K W L\left(V^{3}+3\right)\right)$ \\
\hline
\end{tabular}

\section{Simulation Results and Discussion}

The Monte Carlo simulation results are presented in this section to figure out the performance of the proposed algorithms. The value of $P_{\max }$ is less than $20 \mathrm{dBm}$, whereas the circuit power dissipation at each node is assumed to be $10 \mathrm{dBm}$. The total number of subcarriers, $K$, is set as 5 or 10. The Third-Generation Partnership Project (3GPP) path-loss model is used in our simulations [23]. Additionally, both the Rayleigh fading, $\sim \mathcal{C N}(0,1)$, and the log-normal shadowing effects, $\sim \ln \mathcal{N}(0,8 \mathrm{~dB})$, are considered. The circuit and processing power dissipation values at each node are assumed to be $10 \mathrm{dBm}$, respectively, while the adjacent subcarriers frequency spacing is set to be $12 \mathrm{kHz}$. The thermal noise density is $-174 \mathrm{dBm} / \mathrm{Hz}$. In the proposed EEM algorithm, the value of the maximum number of inner and outer iterations is 10 , while we set the convergence tolerance value as $10^{-5}$. The initial value of the penalty factor $\Psi$ is 0.001 . The distances from the source node to the relay node and from the relay node to the destination and eavesdropper nodes are denoted by $d_{S R}$, $d_{R D}$, and $d_{R E}$, respectively. As benchmarks, we also simulate the performance of the EEM w/o E.

Figure 2 illustrates the convergence behavior of the designed algorithms for a single channel realization with $K=5, d_{S R}=d_{R D}=200 \mathrm{~m}, d_{R E}=300 \mathrm{~m}$, and $P_{\max }=5 \mathrm{dBm}$. It can be observed that the EE performance of the proposed algorithms and also EEM w/o E increases monotonically with the number of iterations, and the iterative algorithms converge within five iterations. 


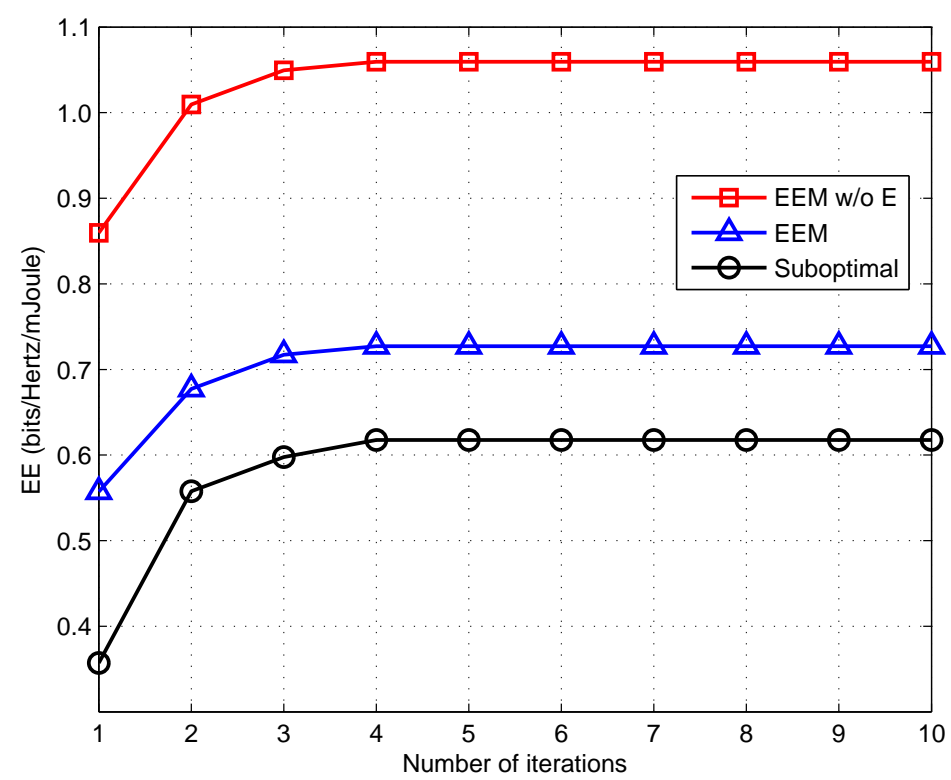

Figure 2. Convergence behavior of the proposed algorithms. EE: energy efficiency; EEM w/o E: EEM algorithm without an eavesdropper.

Figures 3 and 4 show the average EE and SE performance of different algorithms and illustrate the impact of $K$ on the EE and SE performance for $K=5$ and $10, d_{S R}=d_{R D}=200 \mathrm{~m}$, and $d_{R E}=300 \mathrm{~m}$. It can be observed in Figures 3 and 4 that the average EE and SE performance of the algorithms increases significantly as $P_{\max }$ increases, and it remains constant after $P_{\max }=5 \mathrm{dBm}$. Furthermore, when $K$ is increased from 5 to 10 , the average EE is rapidly enhanced because of the better utilization of the available power budget and frequency diversity, while SE decreases due to the increase of the static power consumption. In general, we can observe that when the eavesdropper is active, the performance of the proposed algorithms is worse than that of the EEM w/o E.

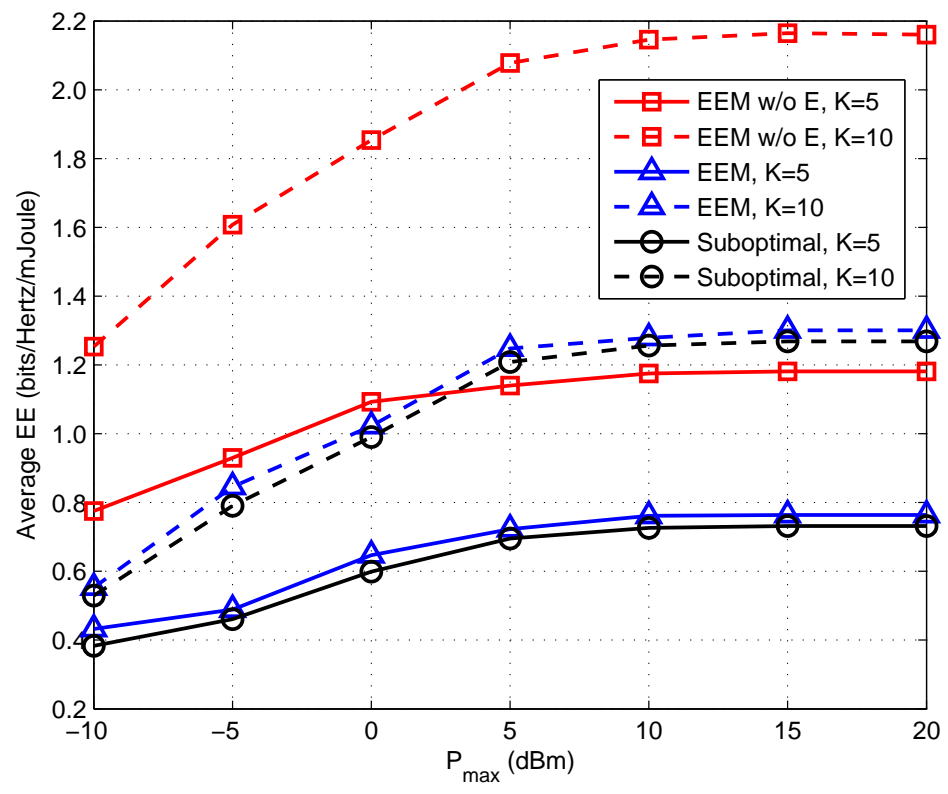

Figure 3. Average EE versus different $P_{\max }$. 


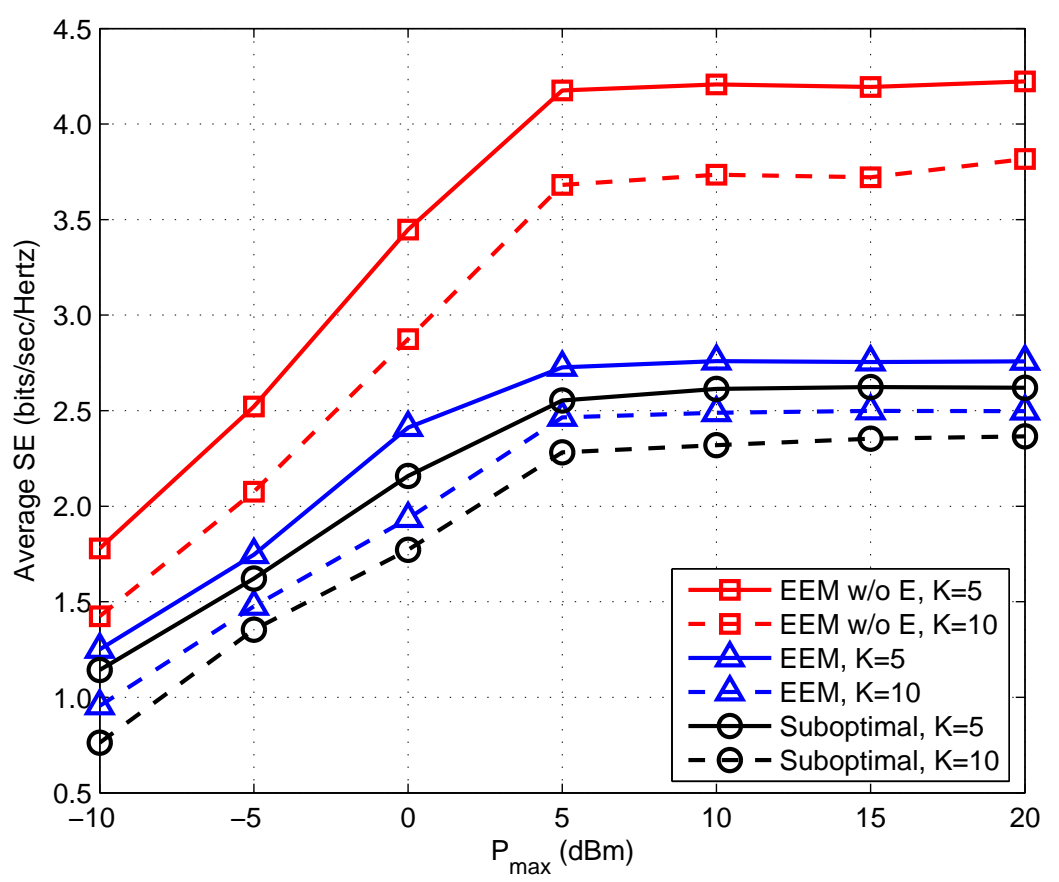

Figure 4. Average spectrum efficiency (SE) versus different $P_{\max }$.

\section{Conclusions}

In this work, we resort to the joint design of subcarrier pairing and power allocation to improve the EE of the DF relay network in the presence of an active eavesdropper. The original problem is highly non-convex. We transform this problem into a convex optimization problem. A dual decomposition-based, iterative algorithm for joint subcarrier pairing and power allocation is investigated for maximizing the EE. A suboptimal algorithm is also investigated to solve the problem with relatively lower complexity. The simulation results demonstrate that the EE performance of the proposed algorithms can be enhanced by increasing $K$ in the joint design.

Acknowledgments: This work was supported by the Ministry of Science and Technology of Taiwan under Grant MOST 106-2221-E-008-014.

Author Contributions: Keshav Singh and Sudip Biswas performed the simulation, analyzed the results and wrote the paper; Meng-Lin Ku and Tharmalingam Ratnarajah proposed the problem and helped in designing the system model and the problem formulation.

Conflicts of Interest: The authors declare no conflict of interest.

\section{References}

1. Chen, Y.; Zhang, S.; Xu, S.; Li, G. Fundamental trade-offs on green wireless networks. IEEE Commun. Mag. 2011, 49, 30-37.

2. Wong, V.W.S.; Schober, R.; Ng, D.W.K.; Wang, L.-C. Key Technologies for 5G Wireless Systems; Cambridge University Press: Cambridge, UK, 2017.

3. Han, C.; Harrold, T.; Armour, S.; Krikidis, I.; Videv, S.; Grant, P.; Haas, H.; Thompson, J.; Ku, I.; Wang, C.-X.; et al. Green radio: Radio techniques to enable energy-efficient networks. IEEE Commun. Mag. 2011, 49, 46-54.

4. Laneman, J.N.; Tse, D.N.C.; Wornell, G.W. Cooperative diversity in wireless networks: Efficient protocols and outage behavior. IEEE Trans. Inf. Theory 2004, 50, 3062-3080. 
5. Arnold, O.; Richter, F.; Fettweis, G.; Blume, O. Power consumption modeling of different base station types in heterogeneous cellular networks. In Proceedings of the Future Network and Mobile Summit 2010, Florence, Italy, 16-18 June 2010; pp. 1-8.

6. Singh, K.; Ku, M.-L. Toward green power allocation in relay-assisted multiuser networks: A pricing-based approach. IEEE Trans. Wirel. Commun. 2015, 14, 2470-2486.

7. Vandendorpe, L.; Duran, R.; Louveaux, J.; Zaidi, A. Power allocation for OFDM transmission with DF relaying. In Proceedings of the IEEE International Conference on Communications, Beijing, China, 19-23 May 2008; pp. 3795-3800.

8. Vandendorpe, L.; Louveaux, J.; Oguz, O.; Zaidi, A. Rate-optimized power allocation for DF-relayed OFDM transmission under sum and individual power constraints. EURASIP J. Wirel. Commun. Netw. 2009, doi:10.1155/2009/814278.

9. Li, W.Y.; Wang, W.; Kong, J.; Peng, M. Subcarrier pairing for amplify-and-forward and decode-and-forward OFDM relay links. IEEE Commun. Lett. 2009, 13, 209-211.

10. Dang, W.; Tao, M.; Mu, H.; Huang, J. Subcarrier-based resource allocation for cooperative multi-relay OFDM systems. IEEE Trans. Wirel. Commun. 2010, 9, 1640-1649.

11. Seong, K.; Mohseni, M.; Cioffi, J.M. Optimal resource allocation for OFDMA downlink systems. In Proceedings of the 2006 IEEE International Symposium on Information Theory, Seattle, WA, USA, 9-14 July 2006; pp. 1394-1398.

12. Ng, D.W.K.; Lo, E.S.; Schober, R. Energy-efficient resource allocation in OFDMA systems with large numbers of base station antennas. IEEE Trans. Wirel. Commun. 2012, 11, 3292-3304.

13. Abedi, M.R.; Mokari, N.; Javan, M.R.; Yanikomeroglu, H. Secure communication in OFDMA-based cognitive radio networks: An incentivized secondary network coexistence approach. IEEE Trans. Veh. Technol. 2017, $66,1171-1185$.

14. Yang, M.; Guo, D.; Huang, Y.; Duong, T.Q.; Zhang, B. Secure multiuser scheduling in downlink dual-hop regenerative relay networks over Nakagami-m fading channels. IEEE Trans. Wirel. Commun. 2016, 15, 8009-8024.

15. El Shafie, A.; Mabrouk, A.; Tourki, K.; Al-Dhahir, N. A secure relay selection AN-aided scheme for dual-hop DF relay networks with two-sided eavesdropping. IEEE Wirel. Commun. Lett. 2017, 6, 474-477.

16. Farhat, J.; Brante, G.; Souza, R.D. On the secure energy efficiency of TAS/MRC with relaying and jamming strategies. IEEE Signal Process. Lett. 2017, 24, 1228-1232.

17. Xiong, J.; Cheng, L.; Ma, D.; Wei, J. Destination-aided cooperative jamming for dual-hop amplify-andforward MIMO untrusted relay systems. IEEE Trans. Veh. Technol. 2016, 65, 7274-7284.

18. Boyd, S.; Vandenberghe, L. Convex Optimization; Cambridge University Press: Cambridge, UK, 2004.

19. Dinkelbach, W. On nonlinear fractional programming. Manag. Sci. 1967, 13, 492-498.

20. Kuhn, H.W. The Hungarian method for the assignment problem. In 50 Years of Integer Programming 1958-2008; Springer: Berlin/Heidelberg, Germany, 2010; pp. 29-47.

21. Zhang, H.; Liu, Y.; Tao, M. Resource allocation with subcarrier pairing in OFDMA two-way relay networks. IEEE Wirel. Commun. Lett. 2012, 1, 61-64.

22. Yu, W.; Lui, R. Dual methods for nonconvex spectrum optimization of multicarrier systems. IEEE Trans. Commun. 2006, 54, 1310-1322.

23. 3rd Generation Partnership Project 3GPP, Further Advancements for E-UTRA Physical Layer Aspects (release 9), 3GPP TR 36.814, V9.0.0. Valbonne, France, 2010. Available online: http:/ /www.qtc.jp/3GPP/ Specs/36814-900.pdf (accessed on 23 November 2017).

(C) 2017 by the authors. Licensee MDPI, Basel, Switzerland. This article is an open access article distributed under the terms and conditions of the Creative Commons Attribution (CC BY) license (http:// creativecommons.org/licenses/by/4.0/). 